\title{
Etude des facteurs favorisant l'impunité a la législation en matière de violences sexuelles en République Démocratique du Congo
}

\author{
Par ILUNGA BONDO Fréddy* et FATU MATA Nadine**
}

\begin{abstract}
RESUME
Les violences sexuelles constituent un véritable problème de santé publique et une cause de violation massive des droits de l'Homme, principalement des femmes et des jeunes enfants. Cette étude s'emploie à repérer les facteurs ou obstacles qui influent négativement sur la punissabilité des auteurs des infractions à la loi $n^{\circ} 06-018$ du 20 juillet 2006 relative aux violences sexuelles en République Démocratique du Congo. Ces facteurs sont de plusieurs ordres, notamment sociologique, culturel, économique et juridique. Il importe de les déceler ou mieux de les dénicher pour permettre aux rouages juridictionnels du pays, qui se veut un "Etat de droit ", de bien rendre la justice par l'application rigoureuse des lois relatives à cette matière de violences sexuelles afin de restaurer les victimes de ces affreux actes dans leur droit et dignité.
\end{abstract}

\section{INTRODUCTION}

L'application de différentes incriminations relatives aux violences sexuelles soulève toute une panoplie d'inquiétudes tant au niveau des tribunaux et de leurs parquets respectifs qu'au niveau de la communauté tout entière. Il est certes vrai que la législation sur les violences sexuelles, en l'occurrence, la loi nº 06-018 du 20 juillet 2006 modifiant et complétant le décret du 30 janvier 1940 portant Code pénal congolais présente plusieurs avantages que nous allons décrire car elle vient combler une lacune créée par de nouvelles formes de violences en même temps qu'elle renforce les droits de la victime à un procès équitable; elle a réduit, ou presque supprimé, quelques barrières qui laissaient impunis certains auteurs des violences sexuelles.

Toutefois, une inquiétude nous hante, l'existence de certaines faiblesses et contraintes liées au contexte actuel rendent difficile son application dans certains cas; parmi elles, on note sa non rétroactivité, l'insuffisance de sa vulgarisation, l'absence d'aide judiciaire en faveur des victimes, l'insécurité dans certaines provinces, le dysfonctionnement du système judiciaire, les racines sociologiques et bien d'autres. Ceci nous pousse à soulever une préoccupation, à savoir, comment faire pour bannir ces faiblesses ou obstacles afin de per-

* ILUNGA BONDO Fréddy, Chef de Travaiux à la Faculté de Droit de l'Université de Kamina.

** Nadine FATUMA MATA, Assistante à l'Institut Supérieur d'Etudes Sociales de Lubumbashi. 
mettre à cette loi de s'appliquer sans tergiversation et de remettre les victimes dans leurs droits constitutionnellement garantis?

La meilleure façon d'appliquer cette loi, serait, par une approche sociojuridique, de déceler avant tout ces faiblesses, de les analyser et de ressortir leur impact éventuel sur l'impunissabilité des auteurs des infractions à la loi sur les violences sexuelles.

Ainsi, pour matérialiser cette étude, nous allons, à l'aide de l'exégèse juridique appuyée par la technique documentaire, d'abord décrire les avantages que présente la législation sur les violences sexuelles(A); ensuite, procéder à un état des lieux des incriminations et à la discussion des résultats récoltés dans le Haut-Lomami (B); enfin, ressortir, sur base de ces données de terrain, les différents facteurs socio-économico-juridiques faisant obstacle à l'application de la législation en matière de violences sexuelles (C).

\section{A. LES AVANTAGES DE LA LÉGISLATION SUR LES VIOLENCES SEXUELLES}

La loi n06-018 du 20 juillet 2006 relatives aux violences sexuelles présente bien des avantages. Parmi ceux-ci, il y a lieu de citer, la prise en compte de nouvelles formes de violences sexuelles, le renforcement des droits de la victime à un procès équitable, le renforcement du régime répressif, le relèvement de l'âge de la majorité pénale.

\section{DE LA PRISE EN COMPTE DE NOUVELLES FORMES DE VIOLENCES SEXUELLES}

La législation a pris en compte de nouvelles formes de violences sexuelles créées par la guerre. C'est dans ce contexte que le législateur congolais a élargi la définition du viol et de l'attentat à la pudeur, l'intégration des infractions empruntées au Droit international humanitaire tels que la grossesse forcée, le mariage forcé, et renforce automatiquement la protection des personnes vulnérables notamment, les femmes et les enfants.

\section{DU RENFORCEMENT DES DROITS DE LA VICTIME A UN PROCES EQUITABLE ET DU REGIME REPRESSIF}

Certains droits procéduraux de la victime ont été renforcés: c'est le cas de la célérité de la procédure, du recours obligatoire au médecin et au psychologue pour évaluer le préjudice, l'intervention du conseil à tous les niveaux de la procédure. Toutes ces règles permettent à la victime d'être mieux défendue et contribuent ainsi à la lutte contre l'impunité. Le régime répressif a aussi été renforcé; désormais, la loi cumule la peine d'emprisonnement et d'amende.

\section{DE LA QUALITE DE L'AUTEUR ET DE L'ORDRE HIERARCHIQUE}

L'article $1^{\text {er }}$ de la loi ${ }^{\circ} 06-018$ du 20 juillet 2006 prévoit l'impertinence de la qualité officielle et de l'ordre hiérarchique en matière d'infractions relatives aux violences sexuelles. A ce propos, elle dispose : « la qualité officielle de l'auteur d'une infraction relative aux vio- 
lences sexuelles ne peut en aucun cas l'exonérer de la responsabilité pénale ni constituer une cause de diminution de la peine. L'ordre hiérarchique ou le commandement d'une autorité légitime, civile ou militaire, n'exonère nullement l'auteur d'une infraction relative aux violences sexuelles de sa responsabilité ».

La qualité de l'auteur se rapportait au rang social que pouvait occuper ce dernier en face de la justice. Autrefois, certaines autorités étaient exonérées de leur responsabilité. Il s'agissait surtout des officiels ou des autorités politico-administratives... qui ne pouvaient pas répondre de leurs actes car ils étaient dispensés de toute responsabilité pénale dans l'exercice de leurs fonctions. Mais, de nos jours, cette qualité n'opère plus car ne pouvant pas exempter l'auteur de violences sexuelles de sa responsabilité et de ce fait, constitue même, aux termes de cette loi, une circonstance aggravante $\left(^{1}\right)$.

Quant à l'ordre hiérarchique, il se déduit également, en quelque sorte, des qualités de l'auteur de violences sexuelles, dans le rapport entre supérieur et subalterne hiérarchiques que l'ordre hiérarchique a autant figuré parmi les causes de justification en matière de violences sexuelles. Mais la nouvelle loi est venue battre en brèche cette justification en rendant l'ordre hiérarchique inopérant $\left({ }^{2}\right)$, traduisant ainsi une avancée significative dans la protection de la personne humaine.

\section{LA FEMME COMME UNIQUE VICTIME}

Le décret du 30 janvier 1940 portant Code pénal congolais n'était élaboré qu'à la faveur de la femme, considérée comme vulnérable et être fragile, seule victime de violences sexuelles, même sur mineur.

Les dispositions du Droit international humanitaire ont considérablement influencé la législation congolaise jusqu'à lui faire prendre en considération tous les deux sexes. L'avant dernier paragraphe de l'exposé des motifs de la loi n 06-018 du 20 juillet 2006 rappelle : «... ainsi, la présente loi modifie et complète le code pénal congolais par l'intégration des règles du Droit humanitaire relatives aux infractions de violences sexuelles. De ce fait, elle prend largement en compte la protection des personnes les plus vulnérables, notamment, les femmes, les enfants et les hommes victimes des infractions de violences sexuelles. Elle contribue ainsi au redressement de la moralité publique, de l'ordre public et de la sécurité dans le pays ». La femme comme l'homme deviennent des victimes des violences sexuelles.

1 Article 171 bis al 3 de la loi n ${ }^{\circ} 06-018$ du 20 juillet 2006 portant violences sexuelles en République Démocratique du Congo.

2 Article 171 bis al 3 de la loi no $06-018$ du 20 juillet 2006 portant violences sexuelles en République Démocratique du Congo. 


\section{L'AGE DE LA VICTIME}

La majorité pénale consacrée par l'article 167 du décret de 1940 était de 14 ans. La personne humaine était menacée dans son bas âge. La loi de 2006 sur les violences sexuelles a relevé cet âge à 18 ans accomplis. Ainsi, avant dix-huit ans, toute personne physique de l'un ou de l'autre sexe est mineure. Tout acte sexuel, de quelque nature que ce soit, avec pareille personne est constitutif d'infraction des violences sexuelles et appelle, sans exonération, la responsabilité pénale de son auteur. La théorie de la dispense d'âge ne peut plus opérer dans le contexte actuel.

Après ce succinct survol des avantages que présente la loi de 2006 sur les violences sexuelles, venons-en, à présent, à l'état des lieux des incriminations et à la discussion des résultats de nos enquêtes de terrain, lesquels résultats pourraient nous amener à dénicher certaines faiblesses qui ont empêché depuis longtemps et empêchent encore cette loi (législation) de s'appliquer correctement. Ces faiblesses, comme nous les examinerons ci-après, sont d'ordre social, juridique, économique...

\section{B. ETAT DES LIEUX DES INCRIMINATIONS DES VIOLENCES SEXUELLES DANS LE HAUT-LOMAMI ET DISCUSSION DES RESULTATS}

Ces données que nous présentons et analysons concernent les années 2007 à juin 2017 auprès des juridictions du Haut-Lomami. Cette recherche n'a pris en considération que les infractions de violences sexuelles perpétrées par les adultes justiciables de ces juridictions, les mineurs en conflit avec la loi relevant, quant à eux, des Tribunaux pour enfants ou Tribunaux de paix, selon le cas.

\section{LES INCRIMINATIONS AU PARQUET PRES LE TRIBUNAL DE GRANDE INSTANCE DE KAMINA}

\begin{tabular}{|l|l|l|l|l|l|l|}
\hline $\mathrm{N}^{\circ}$ & Catégorie & $\begin{array}{l}\text { En fixation } \\
\text { au TGI }\end{array}$ & En cours & $\begin{array}{l}\text { Classé sans } \\
\text { suite }\end{array}$ & $\begin{array}{l}\text { Transmis à } \\
1 \text { 'Auditorat }\end{array}$ & Nombre total \\
\hline 1 & Viol d'enfants & 48 cas & 108 cas & 14 cas & 0 cas & 170 cas \\
\hline 2 & $\begin{array}{l}\text { Viol à l'aide } \\
\text { de violence }\end{array}$ & 11 cas & 26 cas & 2 cas & 1 cas & 40 cas \\
\hline 3 & $\begin{array}{l}\text { Attentat à la } \\
\text { pudeur }\end{array}$ & 0 cas & 6 cas & 0 cas & 0 cas & 6 cas \\
\hline 4 & $\begin{array}{l}\text { Harcèlement } \\
\text { sexuel }\end{array}$ & 0 cas & 5 cas & 0 cas & 0 cas & 5 cas \\
\hline 5 & $\begin{array}{l}\text { Grossesse for- } \\
\text { cée }\end{array}$ & 0 cas & 1 cas & 0 cas & 0 cas & 1 cas \\
\hline 6 & Mariage forcé & 0 cas & 2 cas & 1 cas & 1 cas & 4 cas \\
\hline
\end{tabular}

Sources : données recueillies au secrétariat du parquet de grande instance. 
Ce tableau renseigne bien, pour le viol d'enfants, 170 cas que nous convertissons à $100 \%$. De ce nombre précité, 48 sont en fixation au tribunal représentant ainsi $28.23 \%$; 108 en cours d'instruction donnant ainsi $63.54 \%$ et 14 classés sans suite, faisant ainsi $8.23 \%$.

En ce qui concerne le viol commis à l'aide de violence, il y a eu 40 cas estimés à $100 \%$. De ce nombre se dégage 11 cas en fixation au Tribunal, soit $27.5 \%$; 26, soit $65 \%$ en cours d'instruction et deux cas classés sans suite représentant $5 \%$, ainsi qu'un seul transmis à l'auditorat, soit $2.5 \%$.

Pour l'incrimination d'attentat à la pudeur, le parquet a enregistré 6 cas en cours représentant $100 \%$ de dossiers encore en sa possession.

Pour le harcèlement sexuel, 5 cas étaient en cours d'instruction donnant ainsi $100 \%$.

S'agissant de la grossesse forcée, un seul cas était en cours d'instruction représentant ainsi $100 \%$.

L'infraction de mariage forcé nous a fourni 3 cas dont 2, soit $50 \%$ en cours d'instruction, un seul, soit $25 \%$ classé sans suite et un autre, soit $25 \%$ transmis à l'auditorat militaire.

\section{LES INCRIMINATIONS DE VIOLENCES SEXUELLES AU TRIBUNAL DE GRANDE INSTANCE D KAMINA}

\begin{tabular}{|l|l|l|l|l|l|}
\hline $\mathrm{N}^{\circ}$ & Catégorie & $\begin{array}{l}\text { Cas avec juge- } \\
\text { ment }\end{array}$ & $\begin{array}{l}\text { Cas sans juge- } \\
\text { ment }\end{array}$ & Frappé d'appel & Nombre total \\
\hline 1 & Viol d'enfants & 25 & 69 & 1 & 95 \\
\hline 2 & $\begin{array}{l}\text { Viol à l'aide de } \\
\text { violence }\end{array}$ & 12 & 22 & 0 & 34 \\
\hline 3 & $\begin{array}{l}\text { Attentat à la pu- } \\
\text { deur }\end{array}$ & 22 & 1 & 0 & 3 \\
\hline 4 & $\begin{array}{l}\text { Harcèlement } \\
\text { sexuel }\end{array}$ & 0 & 1 & 0 & 1 \\
\hline 5 & Adultère & 1 & 0 & 0 & 1 \\
\hline 6 & Avortement & 0 & 4 & 0 & 4 \\
\hline 7 & Enlèvement & 3 & 0 & 0 & 3 \\
\hline
\end{tabular}

Sources : données recueillies au greffe du tribunal de grande instance de Kamina.

L'analyse de ces données se conçoit de la manière que voici : 95 cas au total de viol d'enfants ont été enregistrés représentant pour cela $100 \%$. La répartition de ce pourcentage donnerait $26.31 \%$, soit 25 cas déjà jugés; 69 cas attendant le jugement, soit $72.63 \%$ et un seul cas de jugement frappé d'appel, soit $1.05 \%$.

Pour le viol commis à l'aide de violence, 34 cas ont été enregistrés. De ces $100 \%$ de cas, 12 cas, soit $35.3 \%$ ont eu un jugement rendu et 22 , soit $64.7 \%$ attendent encore le jugement. 
Quant à l'attentat à la pudeur, 3cas ont été enregistrés, soit $100 \%$ de cette infraction. De ce pourcentage se dégagent 2 cas avec jugement, soit $66.7 \%$ et un seul, soit $33.3 \%$ dans lequel le juge ne s'est pas encore prononcé.

S'agissant du harcèlement sexuel, un seul cas était en cours d'instruction juridictionnelle, ce qui représentait $100 \%$.

Pour l'enlèvement, trois cas ont été répertoriés en flagrance, jugés avec prononcé de jugement, nous donnant $100 \%$.

Lors de la récolte des données au Tribunal, nous avons trouvé 67 cas de viol d'enfants dont les auteurs étaient eux aussi des mineurs et qui ont été transmis au juge naturel des enfants. Mais par manque des tribunaux pour enfants dans le Haut-Lomami, c'est au Tribunal de paix que tous ces cas ont été déférés.

Nous avons voulu parler de l'avortement ici à cause des conséquences qu'il engendre dans la mesure où il est l'œuvre clandestine des violeurs de mineurs qui cherchent à se disculper en faisant avorter ces mineurs à l'insu de leurs familles. Ceci nous a fourni 4 cas, soit $100 \%$ en cours d'instruction juridictionnelle.

\section{LES INCRIMINATIONS DE VIOLENCES SEXUELLES A L'AUDITORAT ET AU TRIBUNAL MILITAIRE DE GARNISON DU HAUT-LOMAMI}

\begin{tabular}{|l|l|l|l|l|l|l|l|}
\hline$N^{\circ}$ & Catégorie & $\begin{array}{l}\text { En fixation } \\
\text { Au Tribu- } \\
\text { nal M }\end{array}$ & En cours & $\begin{array}{l}\text { Classé } \\
\text { sans suite }\end{array}$ & $\begin{array}{l}\text { Avec juge- } \\
\text { ment }\end{array}$ & $\begin{array}{l}\text { Frappé } \\
\text { d'appel ou } \\
\text { transféré } \\
\text { au civil }\end{array}$ & $\begin{array}{l}\text { Nombre } \\
\text { total }\end{array}$ \\
\hline 1 & $\begin{array}{l}\text { Viol d'en- } \\
\text { fants }\end{array}$ & 0 & 12 & 0 & 0 & 42 & 54 \\
\hline 2 & $\begin{array}{l}\text { Viol à } \\
\text { l'aide de } \\
\text { violence }\end{array}$ & 10 & 5 & 3 & 8 & 2 & 28 \\
\hline 3 & $\begin{array}{l}\text { Attentat à } \\
\text { la pudeur }\end{array}$ & 0 & 0 & 0 & 2 & 0 & 2 \\
\hline 4 & $\begin{array}{l}\text { Harcèle- } \\
\text { ment } \\
\text { sexuel }\end{array}$ & 3 & 2 & 2 & 2 & 0 & 9 \\
\hline 5 & Viol & 11 & 10 & 8 & 25 & 7 & 61 \\
\hline 6 & $\begin{array}{l}\text { Mariage } \\
\text { forcé }\end{array}$ & 0 & 0 & 0 & 1 & 0 & 1 \\
\hline
\end{tabular}

Sources : données recueillies à l'auditorat et au tribunal militaire de garnison de Kamina.

Ces résultats renseignent bien, pour le viol, 61 cas convertis à $100 \%$. De ce pourcentage, 11 cas sont en fixation au tribunal représentant ainsi $18.03 \%$; 10 en cours d'instruction donnant ainsi $16.4 \%$ et 8 classés sans suite, faisant ainsi $13.1 \%$. S'agissant des cas avec jugement, on en a enregistré 25 , soit $40.98 \%$ dont 7 , soit $11.5 \%$ sont frappés d'appel. 
En ce qui concerne le viol commis à l'aide de violence, 28 cas ont été dénombrés représentant les $100 \%$. Ce nombre dégage 10 cas en fixation au Tribunal, soit 35.7\%; 5, soit $17.9 \%$ en cours d'instruction et 3 classés sans suite représentant $10.7 \%$; ainsi que 8 , soit $28.6 \%$ jugés dont 2 , soit $7.1 \%$ frappés d'appel.

Pour ce qui est de viol des mineurs, 54 cas ont été trouvés dont 12, soit $22.2 \%$ étaient en cours d'instruction et 42 , soit $77.8 \%$ étaient déjà transférés au juge naturel pour enfant.

Pour le harcèlement sexuel, l'auditorat a enregistré 9 cas, soit $100 \%$; 3 soit $33.3 \%$ en fixation; 2 soit $22.2 \%$ en cours; 4 autres soit, $44.4 \%$ avec jugement ayant acquis force de chose jugée.

Pour l'incrimination d'attentat à la pudeur, 2 cas étaient en cours d'instruction donnant ainsi $100 \%$.

L'infraction de mariage forcé nous a fourni 1 cas, soit $100 \%$ avec jugement.

Ces données recueillies auprès du tribunal militaire et de l'auditorat près cette juridiction attestent la ferme détermination de ces juridictions à lutter contre l'impunité des auteurs de ces actes ignobles. Nous avons voulu traiter ces données pour ressortir l'ampleur que présente ce fléau de violences sexuelles non seulement chez les populations civiles, mais aussi chez les militaires. Pour rappel, avant la loi n ${ }^{\circ} 06-018$ du 20juillet 2006, le décret de 1940 portant Code pénal devenant obsolète, le Code pénal militaire contenait déjà plusieurs dispositions qui punissaient et érigeaient les violences sexuelles en crimes, cela pour montrer comment la justice militaire protégeait avec acuité la personne humaine.

\section{LES DIFFERENTS FACTEURS SOCIO-ECONOMICO-JURIDIQUES FAISANT OBSTACLE A L'APPLICATION DE LALEGISLATION EN MATIERE DE VIOLENCES SEXUELLES}

\section{DETECTION DES ACTES DE VIOLENCES SEXUELLES}

La prolifération des actes de violences sexuelles sur les personnes vulnérables et surtout sur les enfants est un problème qui implique tout le monde et toutes les instances de protection de droits inhérents à la nature humaine : personnel médical, corps enseignant, éducateur, parents, autorité publique... ces instances devraient en reconnaître les manifestations dans le but d'aider à en repérer les facteurs qui conduisent à l'impunité des violeurs, afin de mieux les combattre et, au besoin, de les éradiquer.

\section{REPERAGE DES CAS DE VIOLENCES SEXUELLES}

1. La nature du problème

Les recherches effectuées et les données recueillies sur les multiples violations de la loi $\mathrm{n}^{\circ}$ 06-018 du 20 juillet 2006 sur les violences sexuelles à l'encontre de personnes vulnérables nous proviennent des instances policières et judiciaires, des services médicaux, des organisations de droits de l'homme, des organisations humanitaires, des associations pour la dé- 
fense des droits de l'enfant et des femmes, les ONGD nationales et internationales luttant contre les violences sexuelles. Ces données ont été récoltées à partir d'une enquête que nous avons menée auprès de ces différents intervenants $\left(^{3}\right)$.

Il est à remarquer que ces données ne sont pas globalisantes. Elles ne révèlent que les violences sexuelles perpétrées contre les personnes physiques vulnérables à partir de l'année 2006, année où la loi nº6-018 du 20 juillet 2006 est entrée en vigueur jusqu'en 2018. Elles sont recueillies auprès de tribunaux civils et militaires de la ville de Kamina.

Il y a lieu d'encourager ici les instances judiciaires dans leur lutte contre les violences sexuelles car un effort inlassable est déployé à ce niveau et beaucoup d'auteurs de violences sexuelles sont appréhendés quand bien même le chiffre noir serait considérable.

Les violences sexuelles entraînent des conséquences multiples à l'égard des victimes. Pour les femmes, elles occasionnent la rupture de l'union conjugale et le rejet par la société. Et pour les enfants, elles constituent la première cause de leur maltraitance. De surcroit, les agresseurs de ces personnes mineures sont le plus souvent des membres de famille soit des assistants sociaux, etc. $\left({ }^{4}\right)$ Et leur nombre croit toujours en dépit de la sensibilisation, et de l'application de la loi de 2006 sur les violences sexuelles. Les victimes les connaissent bien mais se résignent à les dénoncer, ce qui constitue ainsi un facteur d'impunité.

\section{La non dénonciation des cas de violences sexuelles}

L'un des facteurs d'impunité le plus en vue, c'est que les violences sexuelles sont plus rarement dévoilées par les victimes au moment de leur perpétration car ces dernières n'en font cas ou ne révèlent les faits que plusieurs jours, mois ou années plus tard. Et si les parents ne sont pas informés pour faire pression sur les victimes afin de dénoncer, ces cas restent ignorés de la justice et échappent ainsi à la rigueur de loi.

Les révélations claires et spontanées de maltraitance sexuelle sont rares à l'âge de moins de 10 ans. L'enfant dénonce plus facilement la violence sexuelle si une autre victime en signale une similaire commise par le même auteur, si l'agresseur est étranger au cercle familial ou si l'on pose des questions directes au sujet d'agressions éventuelles $\left(^{5}\right)$.

Ce constat se dégage aussi des attitudes des femmes mariées violentées ou agressées sexuellement à l'insu de leurs conjoints; celles-ci se résignent, quelque fois, à dénoncer de peur d'être désapprouvées par les conjoints et rejetées par la communauté. Leurs agresseurs se baladent sans aucune inquiétude se sentant à l'abri de toute poursuite judiciaire. Voilà un autre cas qui empêche la loi de s'appliquer rigoureusement à l'encontre de potentiels criminels.

3 ILUNGA BONDO Freddy, De l'effectivité de la loi nº6-018 du 20 juillet 2006 portant violences sexuelles dans le Haut-Lomami, DEA, Université de Kamina, KAMINA, 2013-2014.

4 MARIE-LAURE, G., et CLAUDINE, M., Les violences sexuelles des mineurs victimes et auteurs: de la parole au soin, Dunod, Paris, 2010, pp11-12.

5 GERARD Lopez, Enfants violés et violentés: le scandale ignoré, Dunod, Paris, 2013, p. 248. 
3. Faut- il croire aux déclarations des victimes mineures?

La société (entendus : la famille, les professionnels) accorde très peu de crédit aux déclarations faites par les enfants au sujet des violences sexuelles. Leur faible niveau de compréhension et leur bas-âge font que les adultes accordent moins d'attention à leurs allégations $\left({ }^{6}\right)$. Pourtant, les allégations mensongères sont extrêmement rares chez les enfants. Ces derniers se résignent même de donner de vraies révélations lorsqu'ils s'estiment rejetés par la société. En sus, des informations utiles, nécessaires à la manifestation de la vérité sur les violences sexuelles sont tues et l'impunité gagne de plus en plus du terrain.

\section{OBSTACLES AU REPERAGE DES VIOLENCES SEXUELLES}

Les difficultés de dépistage et la méconnaissance de la prévalence des violences sexuelles sur les personnes vulnérables dont les femmes et les enfants révèlent des caractéristiques inhérentes aux contextes qui favorisent leur perpétration, notamment des variables propres aux victimes et des facteurs liés aux infracteurs. Parmi ces contextes, les uns sont d'ordre social, religieux, culturel et les autres se situent dans la violence généralisée.

\section{Sur le plan social}

Les violences sexuelles sont souvent méconnues des services compétents en raison du contexte dans lequel elles se déroulent notamment, des réseaux clandestins (proxénétisme, trafic d'humain...), du cadre familial fermé, milieux institutionnels résidentiels (orphelinats, établissement pour enfants handicapés...). Ces victimes vivant le plus souvent à proximité ou avec leur bourreau, dont elles dépendent d'ailleurs pour leur survie, n'ont pas assez de possibilité de les dénoncer pour les violences sexuelles qu'elles ont subies.

\section{Sur le plan religieux}

Le contexte religieux n'est pas, du reste, épargné dans la lutte contre l'impunité des auteurs de violences sexuelles. Il s'avère que les responsables religieux sont devenus actuellement les grands agresseurs sexuels sur les femmes et surtout sur les enfants(le cas récent des prêtres catholique accusés de pédophilie dans certains pays du monde).

Plus l'interdit religieux concernant la sexualité en dehors des liens du mariage est impératif, plus la victime éprouvera des difficultés à dénoncer les faits, de peur qu'elle ne soit expulsée ou suspendue des activités de l'église ou, tout simplement, en vue de protéger le responsable religieux moyennant des garanties convenues avec la victime ou la famille de celle-ci. 


\section{Sur le plan culturel}

Les interdits et les tabous culturels constituent un véritable frein à l'application de la loi sur les violences sexuelles dans la mesure où ils obstruent les révélations d'agressions à caractère sexuel.

Chez les Luba du Kasaï ainsi que chez ceux du Katanga, évoquer la sexualité provoque des sentiments de honte et de gêne pour les interlocuteurs. C'est ainsi que dans bon nombre des cas, les enfants et les femmes ne sont pas encouragées à exprimer leurs difficultés ou à parler en public devant les hommes. De peur d'être désapprouvés, ils se recroquevillent et s'enferment dans un mutisme cachant ainsi des cas de violation de la loi de 2006 sur les violences sexuelles à leurs parents ou maris qui ne le découvrent qu'après avec toutes les conséquences qui s'en suivent.

\section{La non rétroactivité de la loi sur les violences sexuelles}

Ce principe signifie que la loi sur les violences sexuelles ne peut s'appliquer aux faits antérieurement commis avant sa promulgation, obéissant au principe de la non rétroactivité de toute loi.

Par voie de conséquence, tous les actes de violences commis avant le 20 juillet 2006 demeurent impunis, s'il n’y a pas un autre mécanisme spécial pour leur répression.

5. De l'insuffisance de vulgarisation de la législation sur les violences sexuelles

Cette législation n'est pas encore suffisamment vulgarisée à la base en dépit de toutes les tentatives éparses à travers les médias et les organisations de droits de l'homme.

La population congolaise est estimée aujourd'hui à plus de 75 millions d'habitants et les $3 / 5^{\text {ème }}$ vivent en milieu rural où n'est implantée ni chaîne de radiotélévision, ni antenne de communication. Ces populations rurales ignorent pour la plupart d'entre elles, l'existence même de cette loi.

Cette législation a renforcé le système répressif, il faut pour cela que des populations soient avisées afin de savoir à quoi s'en tenir au cas où certains délinquants tomberaient sous le coup de la loi.

\section{L'insécurité dans certaines provinces ou villes et les racines sociologiques}

L'insécurité causée par des troupes armées ou des miliciens et des seigneurs de guerre dans plusieurs endroits du pays en général, et dans le Haut-Lomami en particulier, entraîne des actes de violences sexuelles causés par des hommes en tenue et ces actes se multiplient dans l'impunité. Les cours et tribunaux ne sont pas déployés sinon pas opérationnels dans tous les coins de la République pour y faire face. Dans ce contexte de violence généralisée, engendrée par la guerre et les conflits armés, une agression sexuelle exercée contre les femmes et les enfants semble tolérée. 
S'agissant des racines sociologiques, il est à noter globalement ici la tendance à la banalisation des victimes sexuelles que d'aucuns ont souvent tendance à ramener au niveau de simples faits divers. Plusieurs facteurs spécifiques conduisent à cette dérive, notamment les pesanteurs coutumières.

\section{Les pesanteurs coutumières}

Dans bon nombre de provinces de la République Démocratique du Congo, il existe des coins et recoins où persistent encore des pratiques coutumières qui, indirectement, font l'apologie de certaines formes voilées de violences sexuelles : lévirat, sororat, mariage précoce, l'inceste royal. Nous pouvons citer entre autres :

a) Chez les Tshokwe, dans le Lwalaba au Katanga, les violences sexuelles faites aux enfants sont tolérées (esclavage d'enfants domestiques) et sont considérées comme pratiques acceptables (mariage forcé ou précoce). Chez les Lunda, toujours dans le Lwalaba, la coutume veut que chaque village de l'empire Lunda offre, au début de l'année, une jeune fille vierge au Muant Yav. Celui-ci peut l'introduire dans son harem, si ses performances sexuelles le satisfont.

b) Toujours au Katanga, chez les Luba et Sanga, existe un rituel d'investiture dénommé « inceste royal » $\left(^{7}\right)$ : est un acte hors nature par lequel, le Mulopwe souverain (chez le Luba) et le chef suprême (chez le Sanga), pour renforcer son pouvoir, il est tenu d'avoir des rapports sexuels avec sa propre mère, ses sœurs ou ses proches parents $\left(^{8}\right)$.

c) Au Kasaï occidental, une coutume autorise le jeune frère du défunt à devenir de droit le mari de la veuve;

d) Au Bandundu, dans la tribu Yansi et chez les Mbala, le mariage entre cousins germains est considéré comme licite.

e) Dans la Province Orientale, avec la pratique du NKOKISA, chez les Bowa selon laquelle une jeune fille est livrée comme rétribution à son beau-frère, en compensation de sa grande sœur stérile, ou du sapengbania chez les Zandé où un mari dont la femme est ménopausée est autorisé à prendre en mariage une belle sœur encore en mesure de procréer. Egalement chez les Bagenia où est autorisé le mariage d'une jeune fille à l'issue de son enlèvement, de sa séquestration et de son viol par son futur époux. La pratique dite « ekipemolo » chez les Bowa consiste à favoriser l'accouplement entre une jeune fille et un léopardeau, en guise d'initiation sexuelle;

f) Au Bas Congo, le « kikumbi »est un rite d'initiation sexuelle qui consiste à enfermer des jeunes filles à l'âge de puberté en un lieu isolé où elles sont littéralement prises d'assaut par des soupirants.

7 CRINE MAVAR, B., « Histoire traditionnelle du Shaba » in Revue zaïroise des sciences de l'homme, ONRD, Kinshasa, 1973, pp.35 - 40.

8 KI-ZERBO, J., Histoire de l'Afrique Noire, Hatier, Paris, 1972, p. 82. 
8. Les inégalités sociales à l'égard du jeune garçon et de la jeune fille

Plus les enfants sont jeunes et plus il leur est difficile de traduire leur vécu en quelques mots. En grandissant, ils développent leur capacité à communiquer, à résoudre des problèmes, à prendre des responsabilités... Cependant, certains enfants, parce qu'ils évoluent dans un environnement familial et social précaire ou violent, voient leur épanouissement freiné ou interrompu. C'est le cas notamment, de nombreuses petites filles dans le HautLomami et dans certaines grandes villes du pays où elles sont privées de certains droits.

En effet, en matière de scolarité, les parents aux conditions de vie peu décentes privilégient généralement les fils au détriment des filles car, estiment-ils, les garçons leur seront redevables en les soutenant durant leur vieillesse. Les jeunes filles restent non instruites, analphabètes et se trouvent le plus souvent, avec la complicité de leurs mères qui les envoient dans la rue à la quête de gros poissons capables de subvenir à leurs besoins ménagers, dans l'impossibilité de signaler les agressions sexuelles dont elles sont victimes.

Le peu de considération accordée au genre féminin dont les fonctions sociales traditionnellement reconnues sont la reproduction et le ménage ne se limite pas là, la peur du rejet dans le chef de la jeune fille impubère, la crainte d'être répudiée de la part de l'épouse victime de viol, mais pourtant considérée comme ayant commis l'adultère, sont autant de facteurs entraînant l'impunité de certains actes de violences sexuelles.

9. Intimidation psychologique de la part de l'agresseur et la crainte des représailles par les victimes

Les jeunes victimes sont sensibles aux brimades, aux humiliations, aux sarcasmes, aux mépris, au rejet comme décrit ci-haut, à l'isolement, à l'exclusion et à toutes sortes de privation, au chantage... ils subissent leur sort en silence.

10. Des sentiments de honte et de culpabilité

Les sentiments de honte, de souillure, de culpabilité et de déshonneur censurent les velléités à révéler les agressions subies. Les victimes se sentent d'autant plus fautives qu'elles sont persuadées d'avoir joué un rôle actif dans l'avènement ou le déroulement des faits. Ces sentiments sont renforcés lorsque l'agresseur prétend qu'il n'a fait que céder au comportement provoquant et séducteur de la victime.

\section{La crainte des conséquences de dénonciation et l'honneur des victimes}

De nombreuses conséquences à divers niveaux peuvent accompagner la révélation d'une agression sexuelle subie par une victime, surtout lorsque celle-ci est un enfant. Les victimes en âge d'envisager ces répercussions peuvent les redouter tant pour elles-mêmes que pour leurs familles. Tel est le cas par exemple, du placement en famille d'accueil, la mise sous tutelle du juge d'enfants, le rejet affectif, la colère de la part des membres de la famille, le 
mariage forcé avec l'abuseur, l'honneur de la famille, les représailles de l'abuseur ou de la famille lorsque celui-ci a été appréhendé, jugé et condamné $\left({ }^{9}\right)$.

Dans certaines cultures congolaises, la virginité et la chasteté des jeunes enfants sont un motif de fierté pour la famille qui considère les relations sexuelles prénuptiales comme un déshonneur et une honte aux parents. Dès lors, les agressions sexuelles sont perçues comme une honte et les victimes se sentent déshonorées. Les jeunes filles courant le risque d'être chassées du domicile familial, sont souvent tentées de se suicider ou contraintes d'épouser, bon gré malgré, leurs agresseurs qu'elles n'arrivent toujours pas à dénoncer aux instances judiciaires.

La peur d'un discrédit, d'un déshonneur par l'entourage peut amener les jeunes victimes à taire les cas de violences ou d'en retarder la divulgation. Les victimes peuvent aussi redouter une confrontation avec l'auteur de l'abus sexuel, mieux armés pour la défense. Lorsque ce dernier nie les faits, l'action des victimes risque d'être vexatoire et malveillante. Dès lors, révéler les abus peut apparaître comme plus hasardeux que de les taire.

Les déclarations des victimes mineures concernant les maltraitances ou les agressions dont elles ont été victimes sont parfois confuses et d'ailleurs dans plusieurs causes instruites et jugées par le Tribunal de Grande Instance et les Tribunaux de Paix du Haut-Lomami, des fois, même par le Tribunal Militaire de Garnison, des présumés agresseurs ont été relaxés par manque de lucidité des enfants victimes et de leurs représentants ainsi que pour insuffisance de preuve fournie par le ministère public.

Plus de $60 \%$ des jugements rendus en matière de violences sexuelles par ces juridictions où nous avons assisté comme conseil, dans le cadre de l'exercice de notre profession, ont été soldés par des acquittements des prévenus suite à certains arrangements farfelus entre parties.

\section{Les handicapés, victimes des agressions sexuelles}

Les difficultés des enfants handicapés, à comprendre la situation des violences sexuelles du fait de la carence de leurs capacités cognitives, de la pauvreté de leur imagination, et à les communiquer à cause d'une absence de langage ou de troubles de la personnalité, rendent peu probable la dénonciation des faits $\left({ }^{10}\right)$. En sus, la personne vivant avec handicap s'est toujours résigné à paraître dans certaines circonstances et lorsqu'elle est violentée par l'entourage, la famille; elle s'estime toujours inférieure et prête à tout taire laissant ainsi impuni, l'auteur de ces actes de violences sexuelles.

9 GERARD Lopez, op cit, p. 249.

10 MARCELA Lacub, Le crime était presque sexuel et autres essais de la caustique juridique, Paris, Flammarion, coll. « champs », 2002, pp. 67-70. 


\section{OBSTACLES A LA POURSUITE DES CAS DE VIOLS PAR LES JURIDICTIONS}

La victime de viol qui désire saisir la justice est, la plupart du temps, confrontée à des obstacles. Ceux - ci peuvent être d'ordre général ou liés à l'attitude du personnel judiciaire. Dans certains cas, ces obstacles sont liés à la victime elle - même.

\section{Obstacles liés à l'accès à la justice en général}

Il n'est pas aisé pour la victime d'avoir accès à la justice. Des obstacles d'ordre général s'érigent souvent sur son chemin tels que la pauvreté et l'ignorance du Droit; l'insuffisance et l'éloignement géographique des juridictions; le fait de porter plainte contre inconnu; le manque de confiance dans le système judiciaire ainsi que l'insécurité et la peur des représailles. Aussi la victime doit-elle être courageuse et déterminée pour obtenir justice.

\section{Pauvreté de la plupart des femmes et ignorance du Droit}

La situation des femmes de la République Démocratique du Congo se caractérise au plan économique par la féminisation de la pauvreté $\left({ }^{11}\right)$.Pour assurer la survie de leur famille $\left.{ }^{12}\right)$, elles sont obligées de s'investir dans l'agriculture, l'élevage et dans le secteur informel, à savoir : le petit commerce, la couture, la teinturerie, la poterie, etc.

Mais l'exacerbation des conflits intercommunautaires a souvent aggravé la situation des femmes qui, exposées à des nombreux risques comme le viol et la mort, sont obligées de renoncer à l'exercice de leurs activités $\left({ }^{13}\right)$. D'où l'accroissement du niveau de pauvreté de ces dernières qui ne leur permet pas de payer les frais occasionnés par la justice et se voient confrontées à la difficulté d'avoir accès à la justice pour obtenir réparation $\left({ }^{14}\right)$.

11 Réseau des Femmes pour un Développement Associatif, Réseau des Femmes pour la Défense des Droits et la Paix, International Alert, Le corps des femmes comme champ de bataille durant la guerre en République Démocratique du Congo. Violences sexuelles contre les femmes et les filles au Sud-Kivu (1996-2003), 2004, p. 25, disponible sur http://www.grandslacs.net/doc/4051.pdf. (Consulté le 20 avril 2017).

12 Amnesty International, « République Démocratique du Congo. Violences sexuelles : un urgent besoin des réponses adéquates »,26 octobre 2004, p.39, disponible sur http://www.grandslacs.net/doc /4051.pdf (consulté le 05/04/2019).

13 CIKURU Marie Noël, cité par MOSWA Mombo, L., La répression des infractions se rapportant aux violences sexuelles dans le contexte de crise de la justice congolaise: cas du viol, DEA en Droit, Kinshasa.

14 Global Rights, S.O.S JUSTICE. Quelle justice pour les populations à l'Est de la République Démocratique du Congo?, Rapport d'évaluation du secteur de la justice au Nord et Sud Kivu, Maniema et Nord Katanga, Editions CEDI, août 2005, p.118. 
a) Insuffisance et éloignement géographique des juridictions

L'infraction de viol est de la compétence du Tribunal de Grande Instance. En effet, sa compétence matérielle s'étend à toute infraction punissable d'une peine de 5 ans de servitude pénale à la peine de mort. Pour rappel, l'infraction de viol est punissable de 5 à 20 ans de prison. Lorsque cette dernière a entraîné la mort de la victime, elle est punissable d'une peine de servitude pénale à perpétuité $\left({ }^{15}\right)$.

Cependant le nombre de Tribunaux de Grande Instance dans le pays est insuffisant et territorialement éloignés de certains justiciables, le Haut-Lomami n'en comptant qu'un seul, couvre de longues distances. Ceci démontre combien les victimes de viol n'ont pas accès facile et rapide aux tribunaux, situation qui entraîne le découragement et le recours à une solution coutumière.

b) Plainte contre inconnu

La majorité des victimes de viol commis durant les différents conflits qu'a connus la République Démocratique du Congo ignorent leurs agresseurs. En effet, certains viols ont été commis par des militaires étrangers qui, après la signature des Accord de paix, s'en sont retournés chez eux, échappant ainsi à la justice. D'autres, par contre, ont été commis par plusieurs agresseurs. C'est le cas des viols collectifs où la victime a du mal à reconnaître tous ses agresseurs. Ces différents cas démontrent combien il est difficile pour la victime d'identifier son ou ses agresseurs afin d'obtenir justice. Elle ne peut se résoudre qu'à porter plainte contre " inconnu » avec cette conséquence que « les plaintes contre inconnu sont rarement instruites à moins que le coût de l'instruction soit intégralement supporté par la victime ou une $\mathrm{ONG} »\left({ }^{16}\right)$. Cette attitude des acteurs judiciaires n'encourage nullement la victime à porter plainte et constitue un obstacle qui l'empêche à accéder à la justice.

c) Manque de confiance dans le système judiciaire et recours fréquent au règlement à l'amiable ou coutumier

La justice en République Démocratique du Congo accuse de nombreux dysfonctionnements dus à plusieurs facteurs, notamment, la lenteur du système judiciaire, le coût de la justice et, des fois, la corruption du personnel judiciaire qui fait que « la justice congolaise ne soit plus un service public qui dit la sanction du Droit mais un bien de consommation. N'y accèdent que ceux qui ont les moyens et n'en sont satisfaits que ceux qui en tirent profit » $\left({ }^{17}\right)$. Il en découle un manque de confiance de la part de la victime dans le système judiciaire.

15 Article 170 du Décret du 30 janvier 1940 portant Code pénal congolais.

16 Global Rights, S.O.S JUSTICE. Quelle justice pour les populations à l'Est de la République Démocratique du Congo?, op cit, p.118.

17 Ibidem. 
Par ailleurs, pour pallier le déficit de la justice, la victime du viol préfère recourir aux instances coutumières. Celles - ci sont proches de la victime et utilisent une procédure connue par elle, car puisée dans sa coutume $\left({ }^{18}\right)$. En outre, le recours à celle-ci permet aux parties d'obtenir une réparation du préjudice qu'il est difficile d'obtenir par voie judiciaire suite à l'inexécution des jugements.

\section{Obstacles liés à l'attitude du personnel judiciaire}

Le personnel judiciaire est pour la plupart du temps dubitatif quant à la réunion des moyens de preuve pouvant charger les auteurs d'infractions sur les violences sexuelles. Confronté à la difficulté d'établissement de la preuve, il est obligé d'acquitter les suspects, sur la base de la maxime « in dubio proreo »(le doute profite au prévenu ». Situation qui favorise l'impunité des infracteurs à la loi sur les violences sexuelles $\left({ }^{19}\right)$.

\section{Obstacles liés à la victime du viol}

L'accès à la justice est également obstrué par des obstacles liés à la victime elle - même. Dans la plupart du temps, il s'agit de ceux liés au statut de la femme dans les coutumes de la $\mathrm{RDC}$, à l'atteinte à la dignité de la victime et aux obstacles relatifs à son environnement social.

a) Obstacles liés au statut de la femme dans les coutumes du Congo Démocratique :

Nombreuses coutumes de la République Démocratique du Congo qui, pour certaines ont subi l'influence arabo-musulmane (celles de l'Est) et pour d'autres, encrées dans les us et coutumes congolais (celles du Centre), considèrent la femme comme un être faible qui ne peut revendiquer ses droits même en justice. Du point de vue sexuel, la femme est considérée comme sacrée. Il est interdit de découvrir sa nudité. Une femme dont la nudité a été dévoilée en garde des blessures intérieures dont toute réparation s'avère difficile $\left({ }^{20}\right)$.

\section{b) Obstacles liés à l'atteinte à la dignité de la victime}

La femme victime de viol a du mal à déclarer ce qui lui est arrivé compte tenu du fait que cet acte est un affront qui lui a été fait et qui porte atteinte à sa dignité. Recourir aux cours et tribunaux signifierait pour elle, exposer son intimité et raconter au vu et au su de tous les

18 Idem, p.120.

19 MUNTAZINI Mukimapa T., "Les moyens de preuve à l'épreuve du viol » in Paroles de Justice, Revue de doctrine 2006, Lutte contre les violences sexuelles. RCN Justice et Démocratie, p.79 80.

20 Centre Olame, Service d'écoute et d'accompagnement des femmes traumatisées, les affres de la guerre au Sud Kivu : Le viol, un affront à notre culture et... à la conscience universelle, éditions Olamenka nyanja, Bukavu, 2004, p. 37. 
atrocités qui lui ont été infligées $\left({ }^{21}\right)$. Cet acte n'est pas facile d'autant plus que dans beaucoup de coutumes, parler du sexe est un tabou.

\section{OBSTACLES LIES A L'INTERPRETATION DE LA LOI ELLE-MEME}

La loi $\mathrm{n}^{\circ} 06-018$ du 20 juillet 2006, contient en son sein quatorze incriminations se rapportant aux violences sexuelles dont la plupart d'entre elles sont d'inspiration du Droit occidental. La compréhension de ces incriminations pose problème à cause d'une certaine ambiguïté de contenu. C'est le cas, par exemple, de la grossesse forcée prévue à l'article 174k. En effet, cet article définit la grossesse forcée comme étant le fait de détenir une ou plusieurs femmes rendues enceintes de force ou par ruse.

A bien cerner cette disposition, il s'ensuit qu'il n'y a grossesse forcée que lorsqu'il y a détention de la victime, peu importe la motivation du ravisseur. Ainsi de rapports sexuels non consentis, réalisés en dehors d'un contexte de détention de la victime aboutissant à une grossesse non désirée, ne seront pas constitutifs de l'infraction de grossesse forcée mais du viol.

Les violences sexuelles aux termes des articles 2 et 3 de la loi de 2006 constituent une seule catégorie d'incriminations réparties en 14 infractions. Mais leur lecture fait croire que le législateur en a prévu deux lorsqu'il parle « des infractions de violences sexuelles » et « des autres infractions des violences sexuelles ». Ceci résulterait du fait que le législateur de 2006 a regroupé ensemble les agressions sexuelles (viols) et les atteintes sexuelles (qu'il a appelées autres infractions des violences sexuelles). Cette interprétation faite révèle un sérieux obstacle de nature à rendre l'application de cette loi difficile.

\section{CONCLUSION}

Les infractions sur les violences sexuelles sont des faits terrifiants assimilables à une pandémie qu'il nous faut tous combattre afin de les déraciner et permettre aux victimes de se sentir restaurées dans leurs droits les plus intimes perdus du fait de ces actes affreux et ignobles qu'elles ont endurés dans leur intégrité physique et leur dignité. Beaucoup d'antivaleurs s'érigent en obstacles qui obstruent la meilleure application de la loi relative aux violences sexuelles en République Démocratique du Congo.

Ces obstacles constituent de véritables barrages à l'effectivité de la loi nº6-018 du 20 juillet 2006. L'État devrait les endiguer par le renforcement des mécanismes qui contribueraient à une application correcte de la loi de 2006. En substance, une réadaptation de la loi par sa légère modification s'avérerait nécessaire.

L'amendement de la loi nº6-018 du 20 juillet 2006 par la fusion des articles 2 et 3 pour n'en faire qu'un seul, relatif aux violences sexuelles est un atout pour sa bonne com-

21 NKUWA MILOSI, Georges, «La justice militaire lutte contre les violences sexuelles par la répression » in Paroles de Justice, Revue de doctrine 2006. Lutte contre les violences sexuelles. RCN Justice et Démocratie, p.29. 
préhension. La redéfinition de l'infraction de grossesse forcée en ressortant une définition qui soit globale permettrait sa bonne application. Car l'infraction de grossesse forcée inclut un dol spécial. D'où l'élimination des termes « aura détenu »; de la sorte, lorsque il y a grossesse forcée, l'auteur se verrait poursuivi de grossesse forcée et de viol(ou que l'une constituant une circonstance aggravante de l'autre).

Puisque les violences sexuelles engendrent des conséquences traumatisantes sur les victimes, l'État devrait lutter sérieusement contre ces actes. Pour lutter contre les violences sexuelles et leurs reproductions de proche en proche, de génération en génération, il serait normal de garantir l'égalité de droits, telle que consacrée par la Constitution, entre tous les citoyens d'une part, mais aussi que « les blessures psychiques » des victimes de violences sexuelles et leur réalité « neurobiologique » soient enfin reconnues, comprises et réellement traitées, d'autre part. De la sorte, les droits fondamentaux que l'homme détient de la nature, en tant qu'homme et qui préexistent à toute forme d'organisation sociale, et donc à l'Etat et au Droit positif se verront assurés, car transcendant et s'imposant à eux, et ceux-ci ont justement pour mission d'assurer leur sauvegarde $\left({ }^{22}\right)$.

Il y aurait aussi lieu que l'Etat congolais et les autres partenaires intéressés organisent des activités nécessaires de sensibilisation et de vulgarisation à grande échelle des magistrats, des avocats, des officiers de police judiciaires et des publics ciblés afin que cette loi soit connue à la base, dans tous les coins et recoins de l'arrière-pays.

Les associations des femmes, la société civile et les assemblées provinciales, par les édits, devraient lutter contre les coutumes contra legem et autres pratiques ci-haut décriées. Elles devraient aussi lutter pour l'assistance judiciaire des victimes et leur accompagnement par des assistants sociaux, surtout lorsque celles-ci sont mineurs.

\section{REFERENCES BIBLIOGRAPHIQUES}

1. Loi $\mathrm{n}^{\circ}$ 06-018 du 20 juillet 2006 portant violences sexuelles en République Démocratique du Congo.

10. MARCELA Lacub, Le crime était presque sexuel: et autres essais de la caustique juridique, Paris, coll. « champs », 2002.

11. Réseau des Femmes pour un Développement Associatif, Réseau des Femmes pour la Défense des Droits et la Paix, International Alert, Le corps des femmes comme champ de bataille durant la guerre en République Démocratique du Congo. Violences sexuelles contre les femmes et les filles au Sud-Kivu (1996-2003), 2004, p. 25, disponible sur http://www.grandslacs.net/doc/4051.pdf, co nsulté le 20 avril 2017.

12. Amnesty International, « République Démocratique du Congo. Violences sexuelles : un urgent besoin des réponses adéquates »,26 octobre 2004, p.39, disponible sur http://www.grandslacs.net/doc /4051.pdf consulté le 05/04/2019). 
13. CIKURU Marie Noël, cité par MOSWA Mombo, L., La répression des infractions se rapportant aux violences sexuelles dans le contexte de crise de la justice congolaise: cas du viol, DEA en Droit, Kinshasa.

15. Décret du 30 janvier 1940 portant Code pénal congolais.

16. Global Rights, S.O.S JUSTICE. Quelle justice pour les populations à l'Est de la RDC?, Rapport d'évaluation du secteur de la justice au Nord et Sud Kivu, Maniema et Nord Katanga, Edition CEDI, août 2005 .

19. MUNTAZINI Mukimapa, T., «Les moyens de preuve à l'épreuve du viol » dans Paroles de Justice, Revue de doctrine 2006, Lutte contre les violences sexuelles. RCN Justice et Démocratie.

20. Centre Olame, Service d'écoute et d'accompagnement des femmes traumatisées, les affres de la guerre au Sud Kivu : Le viol, un affront à notre culture et... à la conscience universelle, éditions Olamenka nyanja, Bukavu, 2004

21. NKUWA MILOSI, Georges, « La justice militaire lutte contre les violences sexuelles par la répression » dans Paroles de Justice, Revue de doctrine 2006. Lutte contre les violences sexuelles. RCN Justice et Démocratie.

22. TAMBEDOU Malick, Qu'est-ce que l'Etat de droit, Dakar, RADDHO, Janvier 2004, pp. 24-26.

3. ILUNGA Bondo Freddy, De l'effectivité de la loi nº6-018 du 20 juillet 2006 portant violences sexuelles dans le Haut-Lomami, DEA, Université de Kamina, KAMINA, 2013-2014.

4. MARIE-LAURE, G., et CLAUDINE, M., Les violences sexuelles des mineurs victimes et auteurs: de la parole au soin, Paris, 2010.

5. GERARD Lopez, Enfants violés et violentés: le scandale ignoré, Paris, 2013.

7. CRINE MAVAR, B., "Histoire traditionnelle du Shaba » dans Revue zaïroise des sciences de l'homme, Kinshasa, 1973.

8. KI-ZERBO, J., Histoire de l'Afrique Noire, Paris, 1972. 